Pacific Journal of Mathematic 


\section{THE GENERALIZED INTERVAL TOPOLOGY ON DISTRIBUTIVE LATTICES}

\section{R. H. REDFIELD}

The author has recently introduced the generalized interval topology on a partially ordered set as an alternative to the standard interval topology. In this paper, the structure of generalized segments in lattices is investigated, and sufficient conditions are given for the generalized interval topology on a distributive lattice to be a lattice topology; adding another condition ensures that the topology is Hausdorff. Similar results are obtained for a slight modification of the generalized interval topology, the generalized star-interval topology, and examples are constructed which illustrate less restrictive situations.

1. Introduction; terminology and notation. In [6], we introduced the concept of generalized intervals in a partially ordered set and showed that they could be used in a natural way to define a topology, called the generalized interval topology, on the set. The definition we used was based on one for intervals, which was given by Frink in [4], and which formally extended the "closed set" definition of the usual interval topology on a totally ordered set to an arbitrary partially ordered set. The use of generalized intervals in place of intervals in Frink's definition did not change the topology on unbounded, totally ordered sets; however, on cardinal products of dually (i.e. both upwards and downwards) directed sets, the generalized interval topology turned out to be not only different from Frink's interval topology but in fact precisely the product of the generalized interval topologies on the factors.

In this paper, we investigate the possible continuity of the lattice operations with respect to the generalized interval topology on a distributive lattice, and give conditions which ensure that the topology is Hausdorff. The definition of generalized intervals adds to the corresponding standard interval certain "relatively perpendicular" elements. The motivation for the definition stems from the plane, where one may consider the set

$$
\{(x, y) \mid 1 \leqq y \leqq-1\}
$$

to be an interval rather than the usual set

$$
\{(0, y) \mid 1 \leqq y \leqq-1\}
$$


The standard polar of an $l$-group was used to describe these "relatively perpendicular" elements in [5], and thus pointed the way to the definition in [6] of upper and lower polars in any partially ordered set. To obtain the necessary machinary to ensure that a distributive lattice has a Hausdorff generalized interval topology which is also a lattice topology, we investigate upper and lower polars (\$2) and generalized segments $(\$ 3)$, in some detail. The major results (Theorems 4.3 and 5.2) are proven for certain distributive lattices which, whenever possible, have nontrivial polars that are "minimal" in a natural sense.

Intervals may not be closed with respect to the generalized interval topology. Thus, in [6], we considered the generalized star-interval topology, which for a directed set is just the topology generated by the interval topology and the generalized interval topology. Most of the machinary developed here is valid for star-polars and generalized star-intervals as well as for polars and generalized intervals, and thus only a slight change of hypotheses might be needed to ensure that the main results for the generalized interval topology could be proved directly for the generalized star-interval topology. However, we prefer to use connections, established here and in [6], between the generalized interval and star-interval topologies, to obtain the results for the generalized star-interval topology as corollaries of the results for the generalized interval topology.

Terminology left undefined here may be found in [1], [2], and [9].

Let $(P, \leqq) 1029$ noted a partially ordered set. We use $\vee$ to indicate the least upper bound of two elements, if it exists. A statement of the form $a \vee b=c$ means that $a \vee b$ exists and equals c. We use $\wedge$ (greatest lower bound) similarly. Let $A, B \subseteq$ $P, x, y \in P$. Then

$$
\begin{gathered}
u(A)=\{p \in P \mid p \geqq a \quad \text { for all } a \in A\}, \\
l(A)=\{p \in P \mid p \leqq a \quad \text { for all } a \in A\},
\end{gathered}
$$

$A \wedge B=\{a \wedge b \mid a \in A, b \in B\}, A \vee B=\{a \vee b \mid a \in A, b \in B\}, x \wedge B=$ $\{x\} \wedge B, x \vee B=\{x\} \vee B, u(x, y)=u(\{x, y\})$, and $l(x, y)=l(\{x, y\})$.

We denote an open interval in $P$ by

$$
] a, b[=\{x \in P \mid a<x<b\},
$$

and an interval (or closed interval) by

$$
[a, b]=\{x \in P \mid a \leqq x \leqq b\}
$$

We may combine the notations, as in 


$$
] a, b]=\{x \mid a<x \leqq b\} ; \quad[a, b[=\{x \mid a \leqq x<b\}
$$

An initial segment of $P$ is a set of the form $(-\infty, r]=l(r)$ for some $r \in P$; a final segment of $P$ is a set of the form $[r, \infty)=u(r)$ for some $r \in P$. Frink's interval topology [4] on $P$ takes $P$ and $\phi$, together with all final and initial segments as a subbase for its closed sets. We denote the interval topology on $P$ by $\mathscr{I}(P)$.

Let $\left\{P_{\alpha} \mid \alpha \in A\right\}$ be a collection of partially ordered sets. The cardinal product of the $P_{\alpha}$, denoted by $|\Pi|\left\{P_{\alpha} \mid \alpha \in A\right\}$, is the Cartesian product of the $P$ with order defined pointwise, i.e. by: $f \leqq g$ if and only if $\alpha f \leqq \alpha g$ for all $\alpha \in A$. If $A$ is finite, say $A=\{1,2, \cdots, n\}$, then we usually denote the cardinal product by $P_{1}|\times| P_{2}|\times| \cdots|\times| P_{n}$.

We consistently use totally ordered set to refer to a partially ordered set in which every two elements are comparable. If $P$ is a partially ordered set, and if $T$ is a totally ordered set, then the lexicographic product of $P$ and $T$, denoted by $P \overleftarrow{\times}$, is the product of $P$ and $T$ ordered by: $(a, b) \leqq(p, t)$ if and only if $b<t$, or $b=t$ and $a \leqq p$.

If $G$ is an $l$-group, then for all $A \subseteq G, A^{+}=\{a \in A \mid a \geqq 0\}$ and $A^{-}=\{a \in A \mid a \leqq 0\}$.

We let $N$ be the natural numbers, $Z$ the integers, and $R$ the real numbers. Unless otherwise noted, $N, Z$, and $R$ have their usual orders. By the plane, we mean $R|\times| R$.

If $\mathscr{T}$ is a topology on a set $X$, we use $T_{0}, T_{1}$, and Hausdorff to refer to the corresponding separation axioms in sense of [9]. If $L$ is a lattice with topology $\mathscr{T}$, then $(L, \mathscr{T})$ is a topological lattice if both

$$
\begin{aligned}
& \vee:(L \times L, \mathscr{T} \times \mathscr{T}) \rightarrow(L, \mathscr{T}), \\
& \wedge:(L \times L, \mathscr{T} \times \mathscr{T}) \rightarrow(L, \mathcal{T}),
\end{aligned}
$$

are continuous. Note that $(L, \mathscr{T})$ may be a topological lattice even if $\mathscr{T}$ is not Hausdorff.

2. Upper and lower polars. Upper and lower polars for a partially ordered set were introduced in [6] as a generalization of polars for an $l$-group. For the results of this paper, we need to look into the structure of these new polars more deeply than we did in [6].

Let $(P, \leqq)$ be a partially ordered set. Suppose that $r, s, t \in P$ are such that $r \leqq s \leqq t$. The set

$$
(s, t)_{\top}=\{p \in P \mid p \wedge t=s\}
$$

is called the upper polar of $t$ with respect to $s$ (or the $s, t$ upper polar). The set 


$$
(s, t)_{T}=\{p \in P \mid p \vee r=s\}
$$

is called the lower polar of $r$ with respect to $s$ (or the $s, r$-lower polar).

We noted in [6] that in an $l$-group $G$, for all $g>0$,

$$
\begin{aligned}
(0, g)^{\perp} & =\left(g^{\perp}\right)^{+}, \\
(0,-g) & =\left(g^{\perp}\right)^{-},
\end{aligned}
$$

where $g^{\perp}=\{h \in G|| h \mid \wedge g=0\}$ is the standard polar for an $l$-group (see [3], [8], and [5]). Thus, in the plane,

$$
((0,0),(0,1))^{\perp}=\{(x, 0) \mid x \geqq 0\} .
$$

For $r, s, t \in P$ with $r \leqq s \leqq t$, we define the upper star-polar of $t$ with respect to $s$, denoted by $*(s, t)^{\perp}$, to be $(s, t)^{\perp}$ if $s<t$, and $\{s\}$ if $s=t$. Similarly, the lower star-polar of $r$ with respect to $s$, denoted by $*(s, r)_{\text {, }}$ is defined as $(s, r)$ if $r<s$, and $\{s\}$ if $r=s$. All the results of this section will remain true if polars are replaced by star-polars.

Proposition 2.1. Let $(P, \leqq)$ be a partially ordered set, and let $r, s, t \in P$ be such that $r<s<t$. Then

(i) $(r, t)^{\perp} \subseteq(r, s)^{\perp}$,

(ii) $(t, r) \subseteq \subseteq(t, s)$.

If $(P, \leqq)$ is a modular lattice, then

(iii) $s \vee(r, t)^{\perp} \subseteq(s, t)^{\perp}$,

(iv) $s \wedge(t, r) \uparrow \subseteq(s, r)$.

Proof. (i) Let $b \in(r, t)^{\perp}$. Then $b \wedge t=r$. Clearly $r \leqq b$ and $s \leqq$ $t$. If $u \leqq b$ and $u \leqq s$, then $u \leqq b$ and $u \leqq t$, i.e. $u \leqq b \wedge t=r$. Thus $b \wedge s=r$, i.e. $b \in(r, s)^{\perp}$. Statement (ii) is the dual of (i). (iii) Let $b \in(r, t)^{\perp}$. Then $b \wedge t=r$, and hence

$$
(s \vee b) \wedge t=s \vee(b \wedge t)=s \vee r=s,
$$

i.e. $b \vee s \in(s, t)^{\perp}$. Statement (iv) is the dual of (iii).

Proposition 2.2. Let $(L, \leqq)$ be a distributive lattice. Let $r, s, t \in$ $L$ be such that $r<s \leqq t$. Then the following statements are equivalent :

(i) $\quad r \wedge(t, r)=r \wedge(s, r)$,

(ii) $r \wedge(t, r) \uparrow \supseteq r \wedge(s, r)$,

(iii) $s \wedge(t, r)=(s, r)$,

(iv) $s \wedge(t, r) \uparrow \supseteq(s, r)$. 
Proof. Clearly, if $s=t$, then statements (i) - (iv) are equivalent. Suppose that $s<t$. By Proposition 2.1, (iii) is equivalent to (iv). Clearly by Proposition 2.1,

$$
r \wedge(t, r)_{\uparrow}=r \wedge\left(s \wedge(t, r)_{\uparrow}\right) \subseteq r \wedge(s, r)_{\uparrow} .
$$

Thus (i) is equivalent to (ii). Similarly, one may see that (iv) implies (ii). It remains to show that (ii) implies (iv). Suppose that (ii) holds, and let $x \in(s, r)_{r}$. By (ii), there exists $b \in(t, r)$ r such that $r \wedge b=$ $r \wedge x$. Then $x \vee r=s, b \vee r=t$, and since $t \geqq s \geqq x, t \wedge x=x$. Thus

$$
\begin{aligned}
s \wedge b & =(x \vee r) \wedge b \\
& =(x \wedge b) \vee(r \wedge b) \\
& =(x \wedge b) \vee(r \wedge x) \\
& =x \wedge(b \vee r) \\
& =x \wedge t=x,
\end{aligned}
$$

i.e., $x \in s \wedge(t, r)$. . Therefore, (iv) holds.

Since Proposition 2.2 holds, its dual also holds. We usually will not state the dual of any result explicitly, even though we may use it later on. As an example, however, we will write out the dual of Proposition 2.2:

Proposition 2.3. Let ( $L, \leqq)$ be a distributive lattice. Let $r, s, t \in$ $L$ be such that $r \leqq s<t$. Then the following statments are equivalent.

(i) $t \vee(r, t)^{\perp}=t \vee(s, t)^{\perp}$,

(ii) $t \vee(r, t)^{\perp} \supseteq t \vee(s, t)^{\perp}$,

(iii) $s \vee(r, t)^{\perp}=(s, t)^{\perp}$,

(iv) $s \vee(r, t)^{\perp} \supseteq(s, t)^{\perp}$.

Let $(L, \leqq)$ be a lattice. Let $r, t \in L$ be such that $r<t$. The interval $[r, t]$ has equivalent lower polars if for all $r<s<t$,

$$
s \wedge(t, s)_{\uparrow}=(s, r)_{\uparrow} .
$$

Similarly, $[r, t]$ has equivalent upper polars if for all $r<s<t$,

$$
s \vee(r, s)^{\perp}=(s, t)^{\perp} .
$$

If $[r, s]$ has both equivalent lower polars and equivalent upper polars, then $[r, s]$ is said to have equivalent polars.

We note that in the plane $[r, t]$ has equivalent polars if and only if $[r, t]$ is totally ordered. However, if we let $R^{*}$ be $R$ with $-\infty$ and $\infty$ 
adjoined, and if $L=\left(R^{*}|x| R^{*}\right) \overline{\times} R$, then $[(\infty, \infty,-1),(-\infty,-\infty, 1)]$ has equivalent polars but is not totally ordered. Thus, non-totally ordered intervals may have equivalent polars, even in distributive lattices.

We note that replacing polars by star-polars does not change the above definitions.

Our next result, which will be very useful in the sequal, provides an alternate characterization of intervals which have equivalent lower polars.

Proposition 2.4. Let $(L, \leqq)$ be a distributive lattice. Let $r, t \in$ $L$ be such that $r<t$. Then $[r, t]$ has equivalent lower polars if and only if for all $r \leqq s<t,(t, s)_{\uparrow}=(t, r)_{\uparrow}$, and for all $r<s \leqq t, r \wedge(t, r)_{\uparrow}=$ $r \wedge(s, r)$.

Proof. Suppose the conditions hold, and let $r<s<t$. Since $r \wedge(t, r)_{\uparrow}=r \wedge(s, r)_{\uparrow}$, then $s \wedge(t, r)_{\uparrow}=(s, r)_{\uparrow}$ by Proposition 2.2. Since $(t, s)_{\uparrow}=(t, r)_{\uparrow}$, this implies that $s \wedge(t, s)_{\uparrow}=(s, r)_{\uparrow}$. Therefore, $[r, t]$ has equivalent lower polars. Conversely, suppose that $[r, t]$ has equivalent lower polars. Clearly, it suffices to show that both conditions hold for $r<s<t$. By Proposition 2.1, $(t, s) \Upsilon \supseteq(t, r)$. Let $b \in(t, s)_{\text {r. Then }}$ $s \wedge b \in(s, r)$, since $[r, t]$ has equivalent lower polars, and hence $(s \wedge b) \vee r=s$. Since $(L, \leqq)$ is $s \wedge(b \vee r)=s, s \wedge(b \vee r)=s$, i.e. $b \vee r \geqq$ $s$. Since $b \in(t, s) r, b \vee s=t$. Then

$$
t=b \vee s \leqq b \vee b \vee r=b \vee r \leqq t \vee t=t .
$$

Hence, $b \vee r=t$, i.e. $b \in(t, r)_{\uparrow}$, and therefore, $(t, s)_{\uparrow}=(t, r)_{r}$. For the other condition, we note that, since $[r, t]$ has equivalent lower polars, and since $(t, s)_{\uparrow}=(t, r)_{\uparrow}$ by the above,

$$
s \wedge(t, r)_{\uparrow}=s \wedge(t, s)_{\uparrow}=(s, r)_{\uparrow}
$$

By Proposition 2.2, $r \wedge(t, r) \uparrow=r \wedge(s, r)$.

The last three results of this section will be needed in the sequel.

Proposition 2.5. Let $(L, \leqq)$ be a distributive lattice. Suppose that $r, z, t, d \in L$ are such that $r \leqq z<t \leqq d$ and for all $z<\alpha \leqq d$, $d \in \alpha \vee(z, \alpha)^{\perp}$. If $[r, t]$ has equivalent lower polars, then $[z, t]=\{z, t\}$.

Proof. Suppose that $z<\alpha \leqq t$. Then $z<\alpha \leqq d$, and hence $d \in \alpha \vee(z, \alpha)^{\perp}$. Let $b \in(z, \alpha)^{\perp}$ be such that $d=\alpha \vee b$. Then

$$
t=d \wedge t=(\alpha \vee b) \wedge t=\alpha \vee(b \wedge t) .
$$


If $b \wedge t<t$, then $\alpha \in(t, b \wedge t)_{\top}$. Since $b \in(z, \alpha)^{\perp}, b \geqq z \geqq r$, and hence $b \wedge t \geqq r$. Since $[r, t]$ has equivalent lower polars, this implies (by Proposition 2.4) that $(t, b \wedge t)_{\uparrow}=(t, r)_{\uparrow}$. Thus $\alpha \in(t, r)_{\text {r }}$, i.e. $\alpha=\alpha \vee r=$ $t$. If $b \wedge t=t$, then

$$
z=t \wedge z=t \wedge(b \wedge x)=t \wedge x=\alpha
$$

which contradicts our choice of $\alpha$. Thus $[z, t]=\{z, t\}$.

Proposition 2.6. Let $(L, \leqq)$ be a lattice. Let $k, r, l, t \in L$ be such that $k \leqq r<l<t$ and $[r, t]$ has equivalent lower polars. Then $t \notin l \vee(k, l)^{\perp}$.

Proof. Suppose that $t \in l \vee(k, l)^{\perp}$. Then $t=l \vee b$ for some $b \in(k, l)^{\perp}$, and hence $b \in(t, l)$ Since $[r, t]$ has equivalent lower polars, $l \wedge(t, l)_{\uparrow}=(l, r)_{\uparrow}$, and hence

$$
k=l \wedge b \in l \wedge(t, l)_{\uparrow}=(l, r) \uparrow .
$$

Thus $r=k \vee r=l$, which contradicts our choice of $r$. Therefore, $t \notin l \vee(k, l)^{\perp}$.

Proposition 2.7. Let $(L, \leqq)$ be a distributive lattice. Let $r, u, w, t \in L$ be such that $r \leqq u<w<t$ and $[r, t]$ has equivalent lower polars. Then $t \notin w \vee(u, w)^{\perp}$.

Proof. Suppose $t \in w \vee(u, w)^{\perp}$. Then $t=w \vee b$ for some $b \in(u, w)^{\perp}$. Thus $b \in(t, w) \uparrow$ and hence by Proposition 2.4, $b \in$ $(t, r)_{r}$. Since $b \wedge w=u$,

$$
r=r \wedge u=r \wedge b \wedge w=b \wedge r
$$

i.e., $b \geqq r$. Thus $t=r \vee b=b$. But this imples

$$
u=b \wedge w=t \wedge w=w
$$

which contradicts our choice of $u$ and $w$. Therefore, $t \notin w \vee(u, w)^{\perp}$.

3. Generalized intervals and segments. Let $(P, \leqq)$ be a partially ordered set. Let $r, s, t \in P$ be such that $r \leqq s \leqq t$. Let $[r, s, \infty)$ be the set of points $x \in P$ such that there exists $a \in(s, r)_{\uparrow}$ satisfying
(a) $l(a, r) \neq \phi$
(b) $l(a, r) \subseteq l(x)$. 
Let $(-\infty, s, t]$ be the set of points $y \in P$ such that there exists $b \in(s, t)^{\perp}$ satisfying

(c) $u(b, t) \neq \phi$,

(d) $u(b, t) \subseteq u(y)$.

We note that if $P$ is a lattice, then

$$
\begin{aligned}
(-\infty, s, t] & =\left\{y \in P \mid y \leqq b \vee t \text { for some } b \in(s, t)^{\perp}\right\}, \\
{[r, s, \infty) } & =\{x \in P \mid x \geqq b \wedge r \text { for some } b \in(s, r)\} .
\end{aligned}
$$

Let $[r, s, t]=[r, s, \infty) \cap(-\infty, s, t]$. A generalized final segment of $P$ is a set of the form $[r, s, \infty)$ for $r, s \in P$ with $r \leqq s$; a generalized initial segment of $P$ is a set of the form $(-\infty, s, t]$ for $s, t \in P$ with $s \leqq t$; and a generalized interval of $P$ is a set of the form $[r, s, t]$ for $r, s, t \in P$ with $r \leqq s \leqq t$.

In the plane, the interval $[(0,-1),(0,1)]$ is not a generalized interval; a corresponding generalized interval is

$$
[(0,-1),(0,0),(0,1)]=\{(x, y) \mid-1 \leqq y \leqq 1\}
$$

Let $r, s, t \in P$ be such that $r \leqq s \leqq t$. The sets $*[r, s, \infty)$ and $*(-\infty, s, t]$ are defined in the same way as $[r, s, \infty)$ and $(-\infty, s, t]$ above, except that when polars appear in the definition, they are replaced by the corresponding star-polars. Generalized star-segments and generalized starintervals are defined accordingly. All the results of $\$ 3$ remain true if polars and generalized segments are replaced by the corresponding star-polars and generalzied star-segments.

The following two results are essentially corollaries of Proposition 2.4.

Proposition 3.1. Let $(L, \leqq)$ be a distributive lattice. Let $r, t \in$ $L$ be such that $r<t$ and $[r, t]$ has equivalent lower polars. If $r \leqq s<t$, then $[s, t, \infty) \subseteq[r, t, \infty)$.

Proof. Let $z \in[s, t, \infty)$. Then $z \geqq s \wedge b$ for some $b \in(t, s)_{\text {斤. }}$ Since $[r, t]$ has equivalent lower polars, $b \in(t, r)_{\uparrow}$ by Proposition 2.4. But $z \geqq s \wedge b \geqq r \wedge b$, and hence $z \in[r, t, \infty)$.

PROPOSITION 3.2. Let $(L, \leqq)$ be a distributive lattice. Let $r, s, t \in$ $L$ be such that $r<s \leqq t$ and $[r, t]$ has equivalent lower polars. Then $[r, t, \infty)=[r, s, \infty)$.

Proof. Since $[r, t]$ has equivalent lower polars, by Proposition 2.4

$$
s \wedge(t, r)_{\uparrow}=s \wedge(t, s)_{\uparrow}=(s, r)_{\uparrow} .
$$


Thus

$$
\begin{aligned}
{[r, t, \infty) } & =\left\{y \in L \mid y \geqq b \wedge r \text { for some } b \in(t, r)_{\uparrow}\right\} \\
& =\left\{y \in L \mid y \geqq b \wedge r \wedge s \text { for some } b \in(t, r)_{\uparrow}\right\} \\
& =\left\{y \in L \mid y \geqq c \wedge r \text { for some } c \in(s, r)_{\uparrow}\right\} \\
& =[r, s, \infty)
\end{aligned}
$$

The next result was proven in [7]. Although it will be used only for the main theorem in $\$ 5$, we include it here to enable us to compare Propositions 2.6 and 2.7 with Lemma 3.4.

Proposition 3.3. Let $(L, \leqq)$ be a lattice. Let $r, t \in L$ be such that $r<t$. If $L$ is modular, then

(i) for all $x \in(-\infty, r, t] \cap[t, \infty)$, there exists $b \in(r, t)^{\perp}$ such that $x=t \vee b$.

If $L$ is distributive, then

(ii) for all $x \in(-\infty, r, t] \cap[r, \infty)$, there exists $b \in(r, t)^{\perp}$ such that $x=(x \wedge t) \vee b$.

In view of Proposition 3.3, the following lemma says that if $k=r$ in Proposition 2.6, or if $u=r$ in Proposition 2.7, then we could have assumed that $[r, t]$ had equivalent upper polars instead of equivalent lower polars.

LemmA 3.4. Let $(L, \leqq)$ be a distributive lattice. Let $r<s<t$ be such that $[r, t]$ has equivalent upper polars. Then $t \notin(-\infty, r, s]$.

Proof. Suppose that $t \in(-\infty, r, s$,$] . Then t \leqq s \vee b$ for some $b \in(r, s)^{\perp}$. Since $r<s<t$, then by the dual of Proposition 2.4, $b \in$ $(r, t)^{\perp}$, and hence

$$
t=(s \vee b) \wedge t=s \vee(b \wedge t)=s \vee r=s,
$$

This contradicts our choice of $s$, and thus $t \notin(-\infty, r, s]$.

The next result is the main one of this section, and will be extremely useful in the sequel.

Proposition 3.5. Let $(L, \leqq)$ be a distributive lattice. Let $r, t \in$ $L$ be such that $r<t,[r, t]$ has equivalent upper polars, and $[r, t] \neq\{r, t\}$. Then

$$
\cap\{L \backslash(-\infty, r, s] \mid r<s<t\}
$$

is a dual ideal of $L$. 
Proof. Let $S$ denote $\cap\{L \backslash(-\infty, r, s] \mid r<s<t\}$. If $x \in S$ and $x \leqq y$, then clearly $y \in S$. Suppose that $x, y \in S$, but that $x \wedge y \in$ $(-\infty, r, s]$ for some $s \in L$ with $r<s<t$. Let $x^{\prime}=x \vee r$ and $y^{\prime}=$ $y \vee r$. If $d \in(r, s)^{\perp}$ is such that $x \wedge y \leqq s \vee d$, then

$$
x^{\prime} \wedge y^{\prime}=(x \wedge y) \vee r \leqq s \vee d \vee r=s \vee d .
$$

Thus $x^{\prime} \wedge y^{\prime} \in(-\infty, r, s]$.

If $x^{\prime} \wedge y^{\prime} \geqq t$, then clearly $t \in(-\infty, r, s]$, which contradicts Lemma 3.4. Thus $x^{\prime} \wedge y^{\prime} \supsetneqq t$, and hence, without loss of generality, we may assume that $x^{\prime} \geqq t$. Then $r \leqq x^{\prime} \wedge t<t$. If $r=x^{\prime} \wedge t$, then $x^{\prime} \in(r, t)^{\perp}$. By the dual of Proposition 2.4, $(r, t)^{\perp}=(r, s)^{\perp}$, and thus $x^{\prime} \in(r, s)^{\perp} \subseteq(-\infty, r, s]$. Since $x \leqq x^{\prime}$, this implies that $x \in(-\infty, r, s]$, which contradicts our choice of $x$. Therefore, $r<x^{\prime} \wedge t<t$, and since $[r, t]$ has equivalent upper polars,

$$
\left(x^{\prime} \wedge t\right) \vee\left(r, x^{\prime} \wedge t\right)^{\perp}=\left(x^{\prime} \wedge t, t\right)^{\perp} .
$$

Clearly, $x^{\prime} \in\left(x^{\prime} \wedge t, t\right)^{\perp}$, and hence

$$
x^{\prime} \in\left(x^{\prime} \wedge t\right) \vee\left(r, x^{\prime} \wedge t\right)^{\perp} \subseteq\left(-\infty, r, x^{\prime} \wedge t\right]
$$

Since $x \leqq x^{\prime}, x \in\left(-\infty, r, x^{\prime} \wedge t\right]$, and since $x^{\prime} \wedge t<t$, this contradicts our choice of $x$. We conclude that $x \wedge y \notin(-\infty, r, s]$, and hence that $x \wedge y \in S$. Thus $S$ is a dual ideal of $L$.

The next result, which we will need when we consider the Hausdorff separation axiom, indicates how useful Proposition 3.5 can be.

Proposition 3.6. Let $(L, \leqq)$ be a distributive lattice. Let $r, t \in$ $L$ be such that $r<t$ and $[r, t]$ has equivalent polars. Then

$$
L=(-\infty, r, t] \cup[r, t, \infty) .
$$

Proof. Let $z \in L$. If $(z \wedge t) \vee r=t$, then

$$
z \wedge t \in(t, r) \subseteq[r, t, \infty)
$$

and hence clearly, $z \in[r, t, \infty)$. If $(z \wedge t) \vee r=r$, then $(z \vee r) \wedge t=r$, hence

$$
z \vee r \in(r, t)^{\perp} \subseteq(-\infty, r, t]
$$

and thus clearly, $z \in(-\infty, r, t]$. Otherwise, $r<(z \wedge t) \vee r<t$. Then, in particular, $[r, t] \neq\{r, t\}$, and we may apply Proposition 3.5 to $[r, t]$. Let $T=\cup\{(-\infty, r, s] \mid r<s<t\}$. Since $r<(z \wedge t) \vee r<t$, 


$$
(z \vee r) \wedge t=(z \wedge t) \vee r \in T
$$

Since $t \notin T$ by Lemma 3.4, and since $L \backslash T$ is a dual ideal by Proposition 3.5, $z \vee r \in T$. Since, for all $r<s<t, \quad(-\infty, r, s] \subseteq$ $(-\infty, r, t]$ by the dual of Proposition 3.1, $z \vee r \in(-\infty, r, t]$, and hence $z \in(-\infty, r, t]$.

In some cases, Proposition 3.6 will not give a good enough "separation" of points. Therefore, we must refine it in certain cases to obtain the "separation" described by Proposition 3.8.

LEMMA 3.7. Let $(L, \leqq)$ be a modular lattice. Let $r, s, t, u \in L$ be such that $r<s<t<u,[s, t]=\{s, t\}$, and both $[r, t]$ and $[s, u]$ have equivalent polars. Then

$$
(-\infty, r, t] \backslash(-\infty, r, s] \subseteq[t, u, \infty)
$$

Proof. Let $z \in(-\infty, r, t] \backslash(-\infty, r, s]$. If $(z \wedge t) \vee s=s$, then $(z \vee s) \wedge t=s$ and hence $z \vee s \in(s, t)^{\perp}$. Since $[r, t]$ has equivalent polars,

$$
z \vee s \in s \vee(r, s)^{\perp} \subseteq(-\infty, r, s]
$$

and hence $z \in(-\infty, r, s]$. This contradicts our choice of $z$, and hence $(z \wedge t) \vee s>s$. Since $(z \wedge t) \vee s \leqq t$ and $[s, t]=\{s, t\}$, we must have $(z \wedge t) \vee s=t$, i.e. $z \wedge t \in(t, s)_{\top}$. Since $[s, u]$ has equivalent polars,

$$
z \wedge t \in t \wedge(u, t)_{\uparrow} \subseteq[t, u, \infty)
$$

and therefore, $z \in[t, u, \infty)$.

Proposition 3.8. Let $(L, \leqq)$ be a distributive lattice. Let $r, s, t, u \in L$ be such that $r<s<t<u,[s, t]=\{s, t\}$, and both $[r, t]$ and $[s, u]$ have equivalent polars. Then

$$
L=(-\infty, r, s] \cup[t, u, \infty) .
$$

Proof. Since $[s, t]=\{s, t\}$, clearly $[s, t]$ has equivalent polars. Thus, by Proposition 3.6,

$$
L=(-\infty, s, t) \cup[s, t, \infty) .
$$

By Proposition 3.2 and its dual,

$$
L=(-\infty, r, t] \cup[s, u, \infty)
$$


Therefore, by Lemma 3.7 and its dual,

$$
L=(-\infty, r, s] \cup[t, u, \infty) .
$$

The last result of this section is the "discrete" analog of Proposition 3.5 .

Proposition 3.9. Let $(\mathrm{L}, \leqq)$ be a distributive lattice. Let $r, s, t \in$ $L$ be such that $r<s<t,[r, t]$ has equivalent upper polars, and for all $e \in L$ with $s \leqq e<t, e \in s \vee(r, s)^{\perp}$. Then $L \backslash(-\infty, r, s]$ is a dual ideal of $L$.

Proof. Clearly, if $x \in L \backslash(-\infty, r, s]$ and $x \leqq y$, then $y \in L \backslash(-\infty, r, s]$. Suppose that $x, y \in L \backslash(-\infty, r, s]$, but that $x \wedge y \in(-\infty, r, s]$. Then, similarly to the beginning of the proof of Proposition 3.5, we have that $(x \wedge y) \vee s \in(-\infty, r, s]$, and hence by Lemma 3.4, that

$$
(x \vee s) \wedge(y \vee s)=(x \wedge y) \vee s \not t .
$$

Thus we may assume that $x \vee s \nsupseteq t$, i.e. that $(x \vee s) \wedge t<t$. Then, by hypothesis, $(x \vee s) \wedge t=s \vee b$ for some $b \in(r, s)^{\perp}$. Since $[r, t]$ has equivalent upper polars, this implies that

$$
x \vee s \in((x \vee s) \wedge t, t)^{\perp}=(s \vee b, t)^{\perp}=s \vee b \vee(r, s \vee b)^{\perp} .
$$

If $d \in(r, s \vee b)^{\perp}$, then

$$
r=(s \vee b) \wedge d=(s \wedge d) \vee(b \wedge d),
$$

and hence $s \wedge d \leqq r$. Thus, since $s \wedge b=r$,

$$
s \wedge(b \vee d)=(s \wedge b) \vee(s \wedge d)=r \vee(s \wedge d)=r,
$$

i.e. $\quad b \vee l \in(r, s)^{\perp}$. Therefore, $\quad b \vee(r, s \vee b)^{\perp} \subseteq(r, s)^{\perp}$, and thus $x \vee s \in s \vee(r, s)^{\perp}$. But this implies that $x \vee ; \in(-\infty, r, s]$, and hence that $x \in(-\infty, r, s]$, which contradicts our choice of $x$. We conclude that $x \wedge y \notin(-\infty, r, s]$ and hence that $L \backslash(-\infty, r, s]$ is a dual ideal of $L$.

4. Continuity of the lattice operations. Let $(P, \leqq)$ be a partially ordered set. The generalized interval topology (or gitopology) on $P$, denoted by $\mathscr{G}(P)$, takes as a subbase for its closed sets, $P$ and $\phi$, together with all the final generalized segments and all the 
initial generalized segments. The generalized star-interval topology (or gi-*topology) on $P$, denoted by $G^{*}(P)$ takes as a subbase for its closed sets $P$ and $\phi$, together with all the generalized star-segments. In [6] we proved that $\mathscr{G}(P)$ is an intrinsic topology on $P$, which is preserved by cardinal products of dually directed sets, and that $G^{*}(P)$ is an intrinsic topology which always contains the interval topology, $\mathscr{I}(P)$.

In this section, we show that if intervals with equivalent polars occur throughout a distributive lattice $L$, and if $\mathscr{G}(L)$ is $T_{1}$, then $(L, \mathscr{G}(L))$ is a topological lattice. We first state precisely what is meant by the occurence of intervals with equivalent polars throughout a lattice.

Let $(L, \leqq)$ be a lattice. We say that $r, t \in L$ provide equivalent polars for $x, y, z \in L$ in case $x \leqq r<y<t \leqq z$, and $[r, t]$ has equivalent polars. We say that $L$ has minimal polars if for all $x, y, z \in L$ with $x<y<z$ and $z \notin y \vee(x, y)^{\perp}$, there exist $r, t \in L$ which provide equivalent polars for $x, y, z$. Clearly, replacing polars by star-polars does not change the above definitions. Proposition 5.4 will provide a large class of lattices which have minimal polars.

The first result of this section shows that, for modular lattices, having minimal polars means that whenever $x<y<z$ and there can exist $r, t$ which provide minimal polars for $x, y, z$, then such $r, t$ do in fact exist.

Proposition 4.1. Let $(L, \leqq)$ be a modular lattice. Let $x, y, z, r, t \in L$ be such that $x \leqq r<y<t \leqq z$, and $z \in y \vee(x, y)^{\perp}$. Then $[r, t]$ does not have equivalent lower polars.

Proof. Since $z \in y \vee(x, y)^{\perp}, z=y \vee b$ for some $b \in(x, y)^{\perp}$. Then

$$
\begin{aligned}
& t=z \wedge t=(y \vee b) \wedge t=y \vee(b \wedge t), \\
& x=x \wedge t=(b \wedge y) \wedge t=y \wedge(b \wedge t) .
\end{aligned}
$$

Thus $t \in y \vee(x, y)^{\perp}$. If $[r, t]$ has equivalent lower polars, this contradicts Proposition 2.6.

The following result was noted in [7]. We include it here to indicate that having minimal polars is a self-dual property, i.e. that a lattice has minimal polars if and only if its dual does.

Proposition 4.2. Let $(L, \leqq)$ be a lattice and suppose that $x, y, z \in$ $L$ are such that $x<y<z$. Then the following statments are equivalent :

(i) $z \in y \vee(x, y)^{\perp}$,

(ii) $x \in y \wedge(z, y)$. 
We are now in a position to prove the main result of this section.

THEOREM 4.3. Let $(L, \leqq)$ be a distributive lattice. If $\mathscr{G}(L)$ is $T_{1}$, and if $L$ has minimal polars, the $(L, G(L))$ is a topological lattice.

Proof. Our method is to isolate the difficult part of the proof, and then to prove it separately as Lemma 4.4. We will consider only the continuity of

$$
\wedge:(L \times L, \mathscr{G}(L) \times \mathscr{G}(L)) \rightarrow(L, \mathscr{G}(L))
$$

the continuity of $\vee$ may be proved dually. Since complements of generalized segments form a subbasis for $\mathscr{G}(L)$, it clearly suffices to show that if $x, y \in L$ and $x \wedge y \in L \backslash X$ for some generalized segment $X$, then there exist closed sets $Y$ and $W$ such that $x \in L \backslash W$, $y \in L \backslash Y$, and $(L \backslash W) \wedge(L \backslash Y) \subseteq L \backslash X$.

If $X$ is a generalized final segment, than we may choose $Y$ and $W$ in the following manner. Suppose that $x \wedge y \in L \backslash[k, l, \infty)$ for some $k, l \in L$ with $k \leqq l$. If $\alpha, \beta \in L \backslash[k, l, \infty)$, then $\alpha \wedge \beta \leqq \beta$ and hence $\alpha \wedge \beta \in L \backslash[k, l, \infty)$. Thus, if $x=y$, then

$$
x=x \wedge y=y \in L \backslash[k, l, \infty),
$$

and we may choose $W=[k, l, \infty)=Y$. Suppose $x \neq y$. Clearly either $x \in L \backslash[k, l, \infty)$ or $y \in L \backslash[k, l, \infty)$, and thus, without loss of generality, we may assume that $x \in L \backslash[l, l, \infty)$. Let $Y=\{y\}$ and $W=$ $[k, l, \infty)$. Since $\mathscr{G}(L)$ is $T_{1}, Y$ is closed. If $\alpha \in L \backslash Y$ and $\beta \in L \backslash W$, then $\alpha \wedge \beta \leqq \beta$ and hence $\alpha \wedge \beta \in L \backslash[k, l, \infty)$.

It remains to show that such $Y$ and $W$ exist when $X$ is a generalized initial segment. The problem is more difficult here than in the case where $X$ is a generalized final segment, and requires the hypothesis that $L$ has minimal polars. Suppose that $x \wedge y \in$ $L \backslash(-\infty, k, l]$. Then, since $L$ is a lattice, $k<l$, and hence the proof of Theorem 4.3 will be complete when we prove Lemma 4.4.

LeMmA 4.4. Let $(L, \leqq)$ be a distributive lattice which has minimal polars. If $x, y, k, l \in L$ are such that $k<l$ and $x \wedge y \notin(-\infty, k, l]$, then there exist $u, w \in L$ such that

(i) $x, y \in L \backslash(-\infty, u, w]$;

(ii) for all $\alpha, \beta \in L \backslash(-\infty, u, w], \alpha \wedge \beta \in L \backslash(-\infty, k, l]$.

Proof. We note that since $x \wedge y \notin(-\infty, k, l]$, then $(x \wedge y) \vee l>$ l. Furthermore, if $(x \wedge y) \vee l \in l \vee(k, l)^{\perp}$, then $(x \wedge y) \vee l \in(-\infty, k, l]$, and hence $x \wedge y \in(-\infty, k, l]$. Thus 


$$
(x \wedge y) \vee l \notin l \vee(k, l)^{\perp} .
$$

Also, if $k \leqq r<l$, then

$$
(-\infty, k, l] \subseteq(-\infty, r, l]:
$$

if $z \in(-\infty, k, l]$, then $z \leqq l \vee b$ for some $b \in(k, l)^{\perp}$; by Proposition 2.1 (iii), $r \vee b \in(r, l)^{\perp}$, and since $z \leqq l \vee b=l \vee r \vee b$, this implies that $z \in(-\infty, r, l]$.

(A) Suppose that there exist $c, d \in L$ such that

$$
k \leqq c<l<d \leqq(x \wedge y) \vee l,
$$

$d \notin l \vee(c, l)^{\perp}$, and for all $e \in L$ with $l \leqq e<d, e \in l \vee(c, l)^{\perp}$. Since $L$ has minimal polars, there exist $r, t \in L$ such that $c \leqq r<l<t \leqq d$, and $[r, t]$ has equivalent polars. By Proposition 2.6, $t \notin l \vee(c, l)^{\perp}$, and hence $t=d$. If $l \leqq e<t=d$, then by Proposition 2.1 (iii),

$$
e \in l \vee(c, l)^{\perp}=l \vee\left(r \vee(c, l)^{\perp}\right) \subseteq l \vee(r, l)^{\perp},
$$

and thus by Proposition 3.9, $L \backslash(-\infty, r, l]$ is a dual ideal of $L$.

If $x \wedge y \in(-\infty, r, l]$, then $x \wedge y \leqq l \vee b$ for some $b \in(r, l)^{\perp}$. Thus

$$
t=d \leqq(x \wedge y) \vee l \leqq l \vee b .
$$

Since $b \in(r, l)^{\perp}$ and $[r, t]$ has equivalent polars, $b \in(r, t)^{\perp}$ by the dual of Proposition 2.4. Thus $b \wedge t=r$, and hence

$$
t=(l \vee b) \wedge t=l \vee(b \wedge t)=l \vee r=l .
$$

This contradicts our choice of $t$, and thus $x \wedge y \notin(-\infty, r, l]$. Then clearly, $\quad x, y \in L \backslash(-\infty, r, l]$. Let $\quad \alpha, \beta \in L \backslash(-\infty, r, l]$. Since $L \backslash(-\infty, r, l]$ is a dual ideal, and since $k \leqq c \leqq r<l$, we have by (2) that

$$
\alpha \wedge \beta \in L \backslash(-\infty, r, l] \subseteq L \backslash(-\infty, k, l]
$$

Therefore, if $u=r$ and $w=l$, then conditions (i) and (ii) above are satisfied.

(B) Suppose that for all $c, d \in L$ such that

$$
k \leqq c<l<d \leqq(x \wedge y) \vee l
$$

and $d \notin l \vee(c, l)^{\perp}$, there exists $e \in L$ such that $l<e<d$ and $e \notin l \vee(c, l)^{\perp}$. Since $L$ has minimal polars, by (1) there exist $r, t \in L$ such that 


$$
k \leqq r<l<t \leqq(x \wedge y) \vee l
$$

and $[r, t]$ has equivalent polars. By Proposition 2.6, $t \notin l \vee(r, l)^{\perp}$, and thus, by hypothesis, there exists $t^{\prime} \in L$ such that $l<t^{\prime}<t$ and $t^{\prime} \notin l \vee(r, l)^{\perp}$. Thus, we may find $u, w \in L$ such that $r \leqq u<l<w \leqq t^{\prime}$ and $[u, w]$ has equivalent polars.

Let $r<s<t$. If $s \vee l=t$, then $s \in(t, l)$, and hence by Proposition 2.4, $s \in(t, r)$, i.e. $s=s \vee r=t$. This contradicts our choice of $s$, and thus $s \vee l<t$. Hence, by Lemma 3.4, $t \notin(-\infty, r, s \vee l]$. Suppose that $x \wedge y \in(-\infty, r, s]$. Then by the dual of Proposition 3.1, $x \wedge y \in$ $(-\infty, r, s \vee l]$, and thus, clearly $(x \wedge y) \vee l \in(-\infty, r, s \vee l]$. Since $t \leqq$ $(x \wedge y) \vee l$, this implies that $t \in(-\infty, r, s \vee l]$, which is a contradiction. Thus, $x \wedge y \notin(-\infty, r, s]$. By Lemma 3.4, $t \notin$ $(-\infty, r, s]$. Since $r<s<t$ was arbitrary, we may conclude from Proposition 3.5 that for all $r<s<t$,

$$
x \wedge y \wedge t \notin(-\infty, r, s]
$$

Now suppose that $x \wedge y \wedge t \in(-\infty, u, w]$. Then $x \wedge y \wedge t \leqq w \vee b$ for some $b \in(u, w)^{\perp}$. Hence $x \wedge y \wedge t \in(-\infty, r,(w \vee b) \wedge t]$. If $(w \vee b) \wedge t<t$, this contradicts (3) above. Thus $(w \vee b) \wedge t=t$, i.e. $w \vee(b \wedge t)=t$. Hence $b \wedge t \in(t, w)_{\text {, }}$, and by Proposition 2.4, $b \wedge t \in$ $(t, r)$, i.e. $(b \wedge t) \vee r=t$. Since $b \in(u, w)^{\perp}, b \wedge w=u$. Thus

$$
b \wedge t \geqq b \wedge w=u \geqq r
$$

and hence $(b \wedge t) \vee r=b \wedge t$. But then $b \wedge t=t$, i.e. $b \geqq t$, and we have

$$
u=b \wedge w \geqq t \wedge w=w .
$$

This contradicts our choice of $u$ and $w$, and we conclude $x \wedge y \wedge t \notin$ ($\infty, u, w]$. Clearly, this implies that $x, y \in L \backslash(-\infty, u, w]$. Finally, we note that if $\alpha, \beta \in L \backslash(-\infty, u, w]$, then $\alpha, \beta \in L \backslash(-\infty, u, s]$ for all $u<s<w$ by the dual of Proposition 3.1, and hence $\alpha \wedge \beta \in$ $L \backslash(-\infty, u, s]$ for all $u<s<w$ by Proposition 3.5. Thus, in particular, $\alpha \wedge \beta \in L \backslash(-\infty, u, l]$, and hence by (2), $\alpha \wedge \beta \in L \backslash(-\infty, k, l]$.

Since (A) and (B) exhaust the possibilities, we conclude that Lemma 4.4, and hence Theorem 4.3, hold.

COROllary 4.5. Let $(L, \leqq)$ be a distributive lattice. If $\mathscr{G}(L) \supseteq$ $\mathscr{I}(L)$, and if $L$ has minimal polars, then $(L, \mathscr{G} *(L))$ is a topological lattice. 
Proof. Since $\mathscr{I}(L)$ is $T_{1}, \mathscr{G}(L)$ is $T_{1}$; by [6; Proposition 3.6], $\mathscr{G}(L)=\mathscr{G}^{*}(L)$. The corollary then follows from Theorem 4.3.

We note that proving the results of $\$ 3$ for generalized starsegments, and considering the case of $(-\infty, l]$ in Lemma 4.4 would allow us to drop the hypothesis that $\mathscr{G}(L) \supseteq \mathscr{I}(L)$ in Corollary 4.5 .

5. The Hausdorff separation axiom. This section is devoted primarily to establishing that distributive lattices which have minimal polars, and which satisfy the additional requirement that there be enough polars to "separate" points, have Hausdorff generalized interval topologies.

In [7], we introduced the following condition: A lattice $(L, \leqq)$ is said to be almost polar-dense if, whenever $x, y \in L$ are such that $x<y$ and for all $x<d<y, y \in d \vee(x, d)^{\perp}$, then there exist $c, e \in L$ such that $c<x<y<e, y \notin x \vee(c, x)^{\perp}$, and $x \notin y \wedge(e, y)$. We proved in [7] that a totally ordered set is almost polar-dense if and only if its gi-topology is equivalent to its interval topology (and hence to its gi-*topology). For modular lattices, we have the following [7; Proposition 2.5].

Proposition 5.1. Let $(L, \leqq)$ be a modular lattice. If $L$ is almost polar-dense, then $\mathscr{G}(L)=\mathscr{G}^{*}(L)$.

The main result of this section is the following.

THEOREM 5.2. Let $(L, \leqq)$ be an almost polar-dense, distributive lattice. If $L$ has minimal polars, then $G(L)$ is Hausdorff.

Proof. Let $x, y \in L$ be distinct. Without loss of generality, we may assume that $x<x \vee y$.

(A) Suppose that for all $b, c \in L$ with $x \leqq b<c \leqq x \vee y$, there exists $d \in L$ such that $b<d<c$ and $c \notin d \vee(b, d)^{\perp}$. We first prove the following: $(\alpha)$ If $x \leqq b<c \leqq x \vee y$, then there exist $r, t \in L$ such that $b<r<t<c$ and $[r, t]$ has equivalent polars. To see this, let $x \leqq b<c \leqq x \vee y$. By hypothesis, there exist $d, e, f \in L$ such that $b<d<e<f<c, \quad c \notin f \vee(b, f)^{\perp}, \quad f \notin d \vee(b, d)^{\perp}, \quad$ and $f \notin e \vee(d, e)^{\perp}$. Since $L$ has minimal polars, there exist $r, t \in L$ such that

$$
b<d \leqq r<e<t \leqq f<c,
$$

and $[r, t]$ has equivalent polars. This proves $(\alpha)$.

We first apply $(\alpha)$ to obtain $r, t \in L$ such that $x<r<t<x \vee y$ and $[r, t]$ has equivalent polars. We then apply $(\alpha)$ to $r<t$ to obtain $u, w \in L$ such that $r<u<w<t$ and $[u, w]$ has equivalent polars. By Proposition 3.6, 


$$
L=(-\infty, u, w] \cup[u, w, \infty)
$$

and hence

$$
(L \backslash(-\infty, u, w]) \cap(L \backslash[u, w, \infty))=\phi
$$

By the dual of Proposition 2.7, $r \notin u \wedge(w, u)_{\mid}$. Since $L$ is distributive, this implies that $r \notin[u, w, \infty)$ by the dual of Proposition 3.3. Therefore, $x \in L \backslash[u, w, \infty)$. Dually, $\quad x \vee y \notin(-\infty, u, w]$, and since $x \in$ $(-\infty, u, w]$, this implies that $y \in L \backslash(-\infty, u, w]$.

(B) Suppose that there exist $b, c \in L$ such that $x \leqq b<c \leqq x \vee y$ and for all $b<d<c, c \in d \vee(d, b)^{\perp}$. Since $L$ is almost polar-dense, there exists $k \in L$ such that $k<b$ and $c \notin b \vee(k, b)^{\perp}$. Since $L$ has minimal polars, there exist $r, s \in L$ such that $k \leqq r<b<s \leqq c$ and $[r, s]$ has equivalent polars. By Proposition $2.5,[b, s]=\{b, s\}$, and hence, since $L$ is almost polar-dense, there exists $f \in L$ such that $s<f$ and $f \notin s \vee(b, s)^{\perp}$ (Proposition 4.2). Since $L$ has minimal polars, there exist $u, t \in L$ such that $b \leqq u<s<t \leqq f$ and $[u, t]$ has equivalent polars. Since $[b, s]=\{b, s\}, u=b$, i.e. $[b, t]$ has equivalent polars. By Proposition 3.8,

$$
L=(-\infty, r, b] \cup[s, t, \infty),
$$

and hence

$$
(L \backslash(-\infty, r, b]) \cap(L \backslash[s, t, \infty))=\phi
$$

By the dual of Lemma 3.4, $b \notin[s, t, \infty)$, and therefore, $x \in$ $L \backslash[s, t, \infty)$. Dually, $\quad x \vee y \in L \backslash(-\infty, r, b]$. Since $x \leqq b$, $x \in(-\infty, r, b] \quad$ and thus if $y \in(-\infty, r, b], \quad x \vee y \in$ $(-\infty, r, b]$. Therefore, $y \in L \backslash(-\infty, r, b]$.

We conclude that there exist $\alpha, \beta, \gamma, \delta \in L$ such that $\alpha<\beta, \gamma<\delta$, $y \in L \backslash(-\infty, \alpha, \beta], x \in L \backslash[\gamma, \delta, \infty)$, and

$$
(L \backslash(-\infty, \alpha, \beta]) \cap(L \backslash[\gamma, \delta, \infty))=\phi .
$$

By definition of $\mathscr{G}(L)$,

$$
L \backslash(-\infty, \alpha, \beta], L \backslash(\gamma, \delta, \infty) \in \mathscr{G}(L),
$$

and hence $\mathscr{G}(L)$ is Hausdorff.

COROllary 5.3. Let $(L, \leqq)$ be an almost polar-dense, distributive lattice. If $L$ has minimal polars, then $\mathscr{G}^{*}(L)=\mathscr{G}(L)$ is a Hausdorff lattice topology on $L$. 
Proof. This result follows from Proposition 5.1, Corollary 4.5, and Theorem 5.2.

We conclude this section by showing how to construct many natural examples of almost polar-dense, distributive lattices which have minimal polars.

A partially ordered set $(P, \leqq)$ is said to be unbounded if for all $p \in P$, there exist $r, t \in P$ such that $r<p<t$.

Proposition 5.4. Let $\left\{T_{\alpha} \mid \alpha \in A\right\}$ be a collection of unbounded, totlally ordered sets. Then $|\Pi|\left\{T_{\alpha} \mid \alpha \in A\right\}$ is an almost polar-dense, distributive lattice which has minimal polars.

Proof. By [7; Corollary 2.8], $|\Pi|\left\{T_{\alpha} \mid \alpha \in A\right\}$ is almost polardense. Clearly it is a distributive lattice. That it has minimal polars follows from the fact that if $x, y, z \in|\Pi|\left\{T_{\alpha} \mid \alpha \in A\right\}$ are such that $x<y<z$ and $z \notin y \vee(x, y)^{\perp}$, then there exists $\alpha \in A$ such that $\alpha x<$ $\alpha y<\alpha z$.

6. Some examples. In this section, we construct various examples to illuminate Theorems 4.3 and 5.2 and Corollaries 4.5 and 5.3.

EXAmPle 6.1. Let $M_{5}$ be the five-element nondistributive, modular lattice. Since $\mathscr{G}^{*}\left(M_{5}\right)$ is $T_{1}, \mathscr{G}^{*}\left(M_{5}\right)$ is discrete, and hence a Hausdorff lattice topology. It is easy to see, however, that $\mathscr{G}\left(M_{5}\right)$ is indiscrete since $(-\infty, a, b]=[a, b, \infty)=M_{5}$ for all $a \leqq b$. Thus, $\mathscr{G}\left(M_{5}\right)$ is a lattice topology which is not even $T_{0}$. Clearly, $M_{5}$ is not almost polar-dense, but since if $a<b<c, c \in b \vee(a, b)^{\perp}$, vacuously $L$ has minimal polars.

EXAmPle 6.2. Consider the natural numbers, $N$. Clearly, $\mathscr{G}^{*}(N)$ is discrete, and hence $\left(N, \mathscr{G}^{*}(N)\right)$ is a Hausdorff topological lattice. It was noted in [6] that the closure of $\{1\}$ with respect to $\mathscr{G}(N)$ is $\{1,2\}$, and that therefore, $\mathscr{G}(N)$ is not $T_{1}$. Clearly, $\{n\} \in \mathscr{G}(N)$ for all $n \geqq$ 3 , and furthermore,

$$
\begin{aligned}
\{1,2\} & =L \backslash[3,4, \infty] \in \mathscr{G}(N), \\
\{1\} & =L \backslash[2,3, \infty) \in \mathscr{G}(N) .
\end{aligned}
$$

Since thus $2 \notin\{1\} \in \mathscr{G}(N), \mathscr{G}(N)$ is $T_{0}$. It is easy to see that $(N, \mathscr{G}(N))$ is a topological lattice, and since $N$ is totally ordered, $N$ is distributive. Clearly, $N$ has minimal polars but is not almost polardense. 
EXAmPLE 6.3. Let $L=|\Pi|\{N \mid n \in N\}$. Since the generalized interval topology is preserved by cardinal products of dually directed sets [6], $\mathscr{G}(L)$ is a $T_{0}$, non- $T_{1}$, lattice topology by Example 6.2. We will show first that $\left(L, \mathscr{G}^{*}(L)\right)$ is not a topological lattice, and second that $\mathscr{G} *(L)$ is not Hausdorff.

Let $c_{1}, f \in L$ be defined by $n c_{1}=1$ for all $n \in N$, and

$$
n f=\left\{\begin{array}{ccc}
2 & \text { if } & n=1 \\
1 & \text { if } & n \neq 1
\end{array}\right.
$$

If $a, b, z \in L$ are such that $a \leqq b$ and $z \notin *[a, b, \infty)$, then there exists $l \in N$ such that $l z<l a$ and if $a<b, l a<l b$. We denote the minimal such $l \in N$ by $m(z, a, b)$.

Suppose that $a_{i}, b_{i} c_{j}, d_{j} \in L, 1 \leqq i \leqq \alpha, 1 \leqq j \leqq \beta$, are such that $f \in P_{f}$ where $P_{f} \in G^{*}(L)$ is defined by

(4) $P_{f}=L \backslash\left[\left(\cup\left\{*\left[a_{i}, b_{i}, \infty\right) \mid 1 \leqq i \leqq \alpha\right\}\right) \cup\left(\cup\left\{*\left(-\infty, c_{j}, d_{j}\right] \mid 1 \leqq j \leqq \beta\right\}\right)\right]$

It is easy to see that for all $1 \leqq j \leqq \beta, c_{j}=d_{j}$ and $1 d_{j}=1$. Let $\Gamma \in L$ be defined by

$$
n \Gamma=\left\{\begin{array}{l}
1 \quad \text { if } n=1 \\
1 \text { if } n=m\left(f, a_{i}, b_{i}\right) \text { for some } 1 \leqq i \leqq \alpha \\
\left(\vee\left\{n d_{j} \mid 1 \leqq j \leqq \beta\right\}\right)+1 \quad \text { otherwise }
\end{array}\right.
$$

Since for some $n \in N, n \Gamma=\left(\vee\left\{n d_{j} \mid 1 \leqq j \leqq \beta\right\}\right)+1$, and since $c_{j}=d_{j}$ for all $1 \leqq j \leqq \beta, \Gamma \notin \cup\left\{*\left(-\infty, c_{j}, d_{j}\right] \mid 1 \leqq j \leqq \beta\right\}$. Since

$$
\left(m\left(f, a_{i}, b_{i}\right)\right) \Gamma=1 \leqq\left(m\left(f, a_{i}, b_{i}\right)\right) f<\left(m\left(f, a_{i}, b_{i}\right)\right) a_{i}
$$

for all $1 \leqq i \leqq \alpha, \Gamma \notin \cup\left\{{ }^{*}\left[a_{i}, b_{i}, \infty\right) \mid 1 \leqq i \leqq \alpha\right\}$. Thus $\Gamma \in P_{f}$. Clearly, $\Gamma \wedge f=c_{1}$.

Clearly, $f \in L \backslash\left\{c_{1}\right\} \in \mathscr{G}^{*}(L)$, and $f \wedge f=f$. Thus, if $\left(L, \mathscr{G}^{*}(L)\right)$ is a topological lattice, there exist $P_{1}, P_{2} \in \mathscr{G}^{*}(L)$ such that $f \in P_{1} \cap P_{2}$ and $P_{1} \wedge P_{2} \subseteq L \backslash\left\{c_{1}\right\}$. Since $P_{1}, P_{2} \in \mathscr{G}^{*}(L)$, there exists a $P_{f}$, of the form (4) above, such that $f \in P_{f} \subseteq P_{1} \cap P_{2}$. Then, if $\Gamma$ is constructed as in (5) above,

$$
c_{1}=\Gamma \wedge f \in P_{f} \wedge P_{f} \subseteq P_{1} \wedge P_{2} \subseteq L \backslash\left\{c_{1}\right\}
$$

This is a contradiction, and hence $(L, \mathscr{G} *(L))$ is not a topological lattice.

We conclude this example by showing that $\mathscr{G}^{*}(L)$ is not Hausdorff. Suppose that $c_{1} \in S \in \mathscr{G}^{*}(L)$ and $f \in P \in \mathscr{G}^{*}(L)$. Then, by definition of $\mathscr{G}^{*}(L)$, there exists $P_{f}$, of the form (4) above, such that $f \in P_{f} \subseteq P$, and there exist $r_{k}, s_{k} \in L, 1 \leqq k \leqq \gamma$ such that 


$$
c_{1} \in S_{1}=L \backslash \cup\left\{{ }^{*}\left[r_{k}, s_{k}, \infty\right) \mid 1 \leqq k \leqq \gamma\right\} \subseteq S
$$

Let $Y \in L$ be defined by

$$
n Y=\left\{\begin{array}{l}
1 \quad \text { if } n=m\left(f, a_{i}, b_{i}\right) \text { for some } 1 \leqq i \leqq \alpha \\
1 \quad \text { if } n=m\left(c_{1}, r_{k}, s_{k}\right) \text { for some } 1 \leqq k \leqq \gamma \\
\left(\vee\left\{n d_{j} \mid 1 \leqq j \leqq \beta\right\}\right)+1 \text { otherwise }
\end{array}\right.
$$

As in the case of $\Gamma$ above, $Y \in P_{f}$, and since $m\left(c_{1}, r_{k}, s_{k}\right) Y=1$ for all $1 \leqq k \leqq \gamma, \Upsilon \in S_{1}$. Thus

$$
Y \in P_{f} \cap S_{1} \subseteq P \cap S
$$

i.e. $P \cap S \neq \phi$, and hence $\mathscr{G}^{*}(L)$ is not Hausdorff.

Since $\left[c_{1}, f\right]=\left\{c_{1}, f\right\}$, then for all $c_{1}<d<f, f \in f \vee\left(c_{1}, d\right)^{\perp}$. Thus, since $\left(-\infty, c_{1}\right]=\left\{c_{1}\right\}, L$ is not almost polar-dense. It is easy to see that $L$ has minimal polars.

EXAmple 6.4. Let $L \subseteq R|\times| R$ be defined by

$$
L=([0,2] \times] 0,2]) \cup([3,5] \times[0,2[) \cup\{(0,0),(5,2)\} .
$$

Clearly, $L$ is a lattice. Since the lattice

$$
\{(0,0),(0,1),(1,1),(3,1),(3,0)\}
$$

is a sublattice of $L, L$ is not modular.

We will first show that $\mathscr{G}(L)$ is Hausdorff. Let $(a, b),(x, y) \in L$ be such that $(a, b) \neq(x, y)$. Clearly.

$$
\begin{gathered}
([0,2] \times] 0,2]) \cup\{(0,0)\}=L \backslash[(3,1),(4,1), \infty) \in \mathscr{G}(L), \\
([3,5] \times[0,2[) \cup\{(3,2)\}=L \backslash(-\infty,(1,1),(2,1)] \in \mathscr{G}(L) .
\end{gathered}
$$

Thus, we may assume that $0 \leqq a \leqq 2$ and $0 \leqq x \leqq 2$, or $3 \leqq a \leqq 5$ and $3 \leqq x \leqq 5$. These cases are dual, and hence we will consider only the case where $0 \leqq a \leqq 2$ and $0 \leqq x \leqq 2$. Suppose that $b \neq y$. Without loss of generality, we may assume that $-y<b$. Let $y<\delta<b$. Then $(x, \delta)$, $(a, \delta) \in L$, and

$$
\begin{gathered}
(L \backslash(-\infty,(x, y),(x, \delta)]) \cap(L \backslash[(a, \delta),(a, b), \infty))=\phi \\
(a, b) \in L \backslash(-\infty,(x, y),(x, \delta)] \in \mathscr{G}(L), \\
(x, y) \in L \backslash[a, \delta),(a, b), \infty) \in \mathscr{G}(L)
\end{gathered}
$$


If $b=y$, then we may assume that $x<a$. Since $b=y, 0<y$. Thus, if $x<\gamma<a,(\gamma, b)=(\gamma, y) \in L$, and

$$
\begin{gathered}
(L \backslash(-\infty,(x, y),(\gamma, y)]) \cap(L \backslash[(\gamma, b),(a, b), \infty))=\phi, \\
(a, b) \in L \backslash(-\infty,(x, y),(\gamma, y)] \in \mathscr{G}(L), \\
(x, y) \in L \backslash[(\gamma, b),(a, b), \infty) \in \mathscr{G}(L) .
\end{gathered}
$$

We conclude that $\mathscr{G}(L)$ is Hausdorff.

We will show next that $(L, \mathscr{G}(L))$ is not a topological lattice. Suppose that $(3,0) \notin(-\infty, x, y]$ for some $x \leqq y$. Then clearly,

$$
[(3,0),(3,1)] \subseteq L \backslash(-\infty, x, y]
$$

Suppose that $(3,0) \notin[(a, b),(c, d), \infty)$ for $(a, b) \leqq(c, d)$. If $b=0$, then $3<a<c$, and thus,

$$
[(3,0),(3,1)] \subseteq L \backslash[(a, b),(c, d), \infty)
$$

If $b>0$, then for $0<\eta<b$,

$$
[(3,0),(3, \eta)] \subseteq L \backslash[(a, b),(c, d), \infty)
$$

Let $Y=L \backslash[(1,1),(2,1), \infty)$. Then $(0,0) \in Y \in \mathscr{G}(L)$. We note that $(2,1) \wedge(3,0)=(0,0)$. If $(2,1) \in A \in \mathscr{G}(L)$ and $(3,0) \in B \in \mathscr{G}(L)$, then we wish to show that $A \wedge B \not \subset$. By definition of $\mathscr{G}(L)$,

$$
(3,0) \in \bigcap_{i=1}^{n}\left(L \backslash X_{i}\right) \subseteq B,
$$

where the $X_{i}$ are generalized initial and final segments. By (6), (7), and (8), there exists $0<\mu<1$ such that

$$
[(3,0),(3, \mu)] \subseteq \bigcap_{i=1}^{n}\left(L \backslash X_{i}\right) \subseteq B
$$

Thus, we have $(2,1) \in A$ and $(3, \mu) \in B$, and hence $(2, \mu) \in$ $A \wedge B$. Clearly

$$
(2, \mu) \in[(1,1),(2,1), \infty)
$$

and hence $(2, \mu) \notin Y$. Thus, $A \wedge B \nsubseteq \subset$, and since $(2,1) \wedge(3,0)=(0,0)$, we therefore conclude that 


$$
\wedge:(L \times L, \mathscr{G}(L) \times \mathscr{G}(L)) \rightarrow(L, \mathscr{G}(L))
$$

is not continuous. Hence, $(L, \mathscr{G}(L))$ is not a topological lattice. We note that dually one may show that $v$ is also not continuous.

We show next that $\mathscr{G}(L)=\mathscr{G}^{*}(L)$. Clearly, if $(a, b) \in L$ is such that $0<a$ and $0<b$, then there exists $(c, d) \in L$ such that

$$
(-\infty,(a, b)]=(-\infty,(c, d),(a, b)] .
$$

Furthermore, it is easy to see that if $x>0$,

$$
\begin{gathered}
(-\infty,(0, x)]=(-\infty,(0,0),(0, x)] \cap\left(\bigcap_{n \in N}\left(-\infty,\left((n+1)^{-1}, 1\right),\left(n^{-1}, 1\right)\right]\right), \\
(-\infty,(x, 0)]=(-\infty,(0,0),(x, 0)] \cap\left(\bigcap_{n \in N}\left(-\infty,\left(0,(n+1)^{-1}\right),\left(0, n^{-1}\right)\right]\right), \\
(-\infty,(0,0)]=\bigcap_{n \in N}\left(-\infty,\left((n+1)^{-1},(n+1)^{-1}\right),\left(n^{-1}, n^{-1}\right)\right] .
\end{gathered}
$$

Similarly, final segments are closed with respect to $\mathscr{G}(L)$, and hence $\mathscr{I}(L) \subseteq \mathscr{G}(L)$. Since $L$ is a lattice, this implies that $\mathscr{G}^{*}(L)=\mathscr{G}(L)$ by [6; Proposition 3.6]. We conclude that $G^{*}(L)$ is Hausdorff, but that $\left(L, \mathscr{G}^{*}(L)\right)$ is not a topological lattice.

Since $[(0,0),(3,0)]=\{(0,0),(3,0)\}$, and since $(-\infty,(0,0)]=$ $\{(0,0)\}$, then $L$ is not almost polar-dense. Furthermore, if $3<t \leqq 4$, then

$$
\begin{aligned}
((0,0),(3,0))^{\perp} & =([0,2] \times] 0,2]) \cup\{(0,0)\}, \\
((3,0),(t, 0))^{\perp} & =[(3,0),(3,2)[.
\end{aligned}
$$

Thus, $(4,0) \notin((0,0),(3,0))^{\perp}$, and if

$$
(0,0) \leqq(r, 0)<(3,0)<(t, 0) \leqq(4,0),
$$

then

$$
\begin{aligned}
(3,0) \vee((r, 0),(3,0))^{\perp} & =(3,0) \vee((0,0),(3,0))^{\perp} \\
& =[(3,0),(3,2)[\cup\{(5,2)\} \\
& \neq((3,0),(t, 0))^{\perp}
\end{aligned}
$$

Therefore, $[(r, 0),(t, 0)]$ does not have equivalent polars, and we conclude that $L$ does not have minimal polars. 
Added in Proof. Theorem 4.3 does not require the hypothesis that $\mathscr{G}(L)$ is $T_{1}$ : Let $y=\varnothing$ instead of $\{y\}$ in the second part of the second paragraph of the proof.

\section{REFERENCES}

1. G. Birkhoff, Lattice Theory, third edition, Amer. Math. Soc. Coll. Pub. 25, Providence, 1967.

2. N. Bourbaki, General Topology, Addison-Wesley Pub. Co., Don Mills, Ontario, 1966 (translated from the French, Topologie Générale, Hermann, Paris).

3. P. Conrad, Lex-subgroups of lattice-ordered groups, Czech. Math. J. 18 (1968, 86 - 103.

4. O. Frink, Topology in lattices, Trans. Amer. Math. soc., 51 (1942), $569-582$.

5. R. H. Redfield, A topology for a lattice-ordered group, Trans. Amer. Math. Soc. 187 (1974), $103-125$.

6. - Generalized intervals and topology, (to appear).

7. - Almost polar-dense lattices, Canad. Math. Bull., (to appear).

8. F. Šik, Zur theorie der holbgeordnete Gruppen, Czech. Math. J., 6 (1956), $1-25$.

9. W. Thron, Topological Structures, Holt, Rinehart and Winston, New York, 1966.

Received October 15, 1973.

SIMON FRASER UNIVERSITY

Present address: MONASH UNIVERSITY 


\section{CONTENTS}

Zvi Artstein and John A. Burns, Integration of compact set-valued functions

J. A. Beachy and W. D. Blair, Rings whose faithful left ideals are cofaithful

Mark Benard, Characters and Schur indices of the unitary reflection group $[321]^{3}$

H. L. Bentley and B. J. Taylor, Wallman rings ............................. 15

E. Berman, Matrix rings over polynomial identity rings II ............... 37

Simeon M. Berman, A new characterization of characteristic functions of absolutely continuous distributions ........................ 323

Monte B. Boisen, Jr. and Philip B. Sheldon, Pre-Prüfer rings ............ 331

A. K. Boyle and K. R. Goodearl, Rings over which certain modules are injective

J. L. Brenner, R. M. Crabwell and J. Riddell, Covering theorems for finite nonabelian simple groups. $V$

H. H. Brungs, Three questions on duo rings .................................... 345

Iracema M. Bund, Birnbaum-Orlicz spaces of functions on groups ....351

John D. Elwin and Donald R. Short, Branched immersions between 2-manifolds of higher topological type

J. K. Finch, The single valued extension property on a Banach space ................................................................................. 61

J. R. Fisher, A Goldie theorem for differentiably prime rings ............ 71

Eric M. Friedlander, Extension functions for rank 2, torsion free abelian groups ...................................................................... 371

J. Froemke and R. Quackenbusch, The spectrum of an equational class of groupoids

B. J. Gardner, Radicals of supplementary semilattice sums of

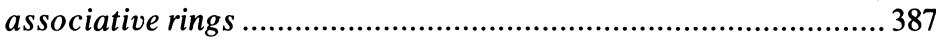

Shmuel Glasner, Relatively invariant measures ...............................393

G. R. Gordh, Jr. and Sibe Mardešić, Characterizing local connectedness in inverse limits...

S. Graf, On the existence of strong liftings in second countable topological spaces

S. Gudder and D. Strawther, Orthogonally additive and orthogonally increasing functions on vector spaces ........................................427

F. Hansen, On one-sided prime ideals .......................................... 79

D. J. Hartfiel and C. J. Maxson, A characterization of the maximal monoids and maximal groups in $\beta x$.

Robert E. Hartwig and S. Brent Morris, The universal flip matrix and the generalized faro-shuffle 


\section{Pacific Journal of Mathematics}

Vol. 58, No. 1

March, 1975

John Allen Beachy and William David Blair, Rings whose faithful left ideals are cofaithful .................................... 1

Herschel Lamar Bentley and Barbara June Taylor, Wallman rings ........ 15

Elizabeth Berman, Matrix rings over polynomial identity rings. II ...... 37

Ann K. Boyle and Kenneth R. Goodearl, Rings over which certain modules are injective ................................. 43

J. L. Brenner, Robert Myrl Cranwell and James Riddell, Covering theorems for finite nonabelian simple groups. $V \ldots \ldots \ldots \ldots \ldots \ldots \ldots \ldots \ldots$

James Kenneth Finch, The single valued extension property on a Banach

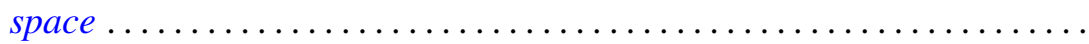

John Robert Fisher, A Goldie theorem for differentiably prime rings........ Friedhelm Hansen, On one-sided prime ideals .................... Jon Craig Helton, Product integrals and the solution of integral equations..........................................

Barry E. Johnson and James Patrick Williams, The range of a normal

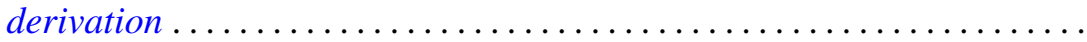

Kurt Kreith, A dynamical criterion for conjugate points ............ 123

Robert Allen McCoy, Baire spaces and hyperspaces .............. 133

John McDonald, Isometries of the disk algebra ................ 143

H. Minc, Doubly stochastic matrices with minimal permanents ......... 155

Shahbaz Noorvash, Covering the vertices of a graph by vertex-disjoint paths. ...

Theodore Windle Palmer, Jordan *-homomorphisms between reduced Banach*-algebras

Donald Steven Passman, On the semisimplicity of group rings of some

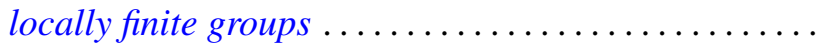

Mario Petrich, Varieties of orthodox bands of groups .

Robert Horace Redfield, The generalized interval topology on distributive

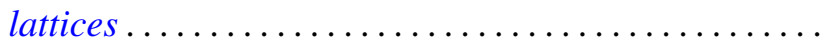

James Wilson Stepp, Algebraic maximal semilattices .... . .

Patrick Noble Stewart, A sheaf theoretic representation of rings with Boolean orthogonalities ........................

Ting-On To and Kai Wing Yip, A generalized Jensen's inequality......... 255

Arnold Lewis Villone, Second order differential operators with self-adjoint

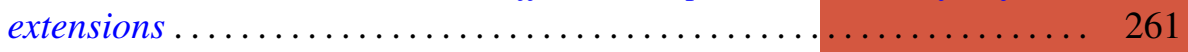

Martin E. Walter, On the structure of the Fourier-Stieltjes algebra ....... 267

John Wermer, Subharmonicity and hulls .................... 283

Edythe Parker Woodruff, A map of $E^{3}$ onto $E^{3}$ taking no disk onto a

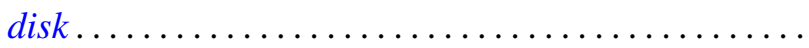

